\title{
Variants of Olfactory Memory and Their Dependencies on the Hippocampal Formation
}

\author{
Ursula Stäubli, ${ }^{1}$ To-Tam Le, ${ }^{2}$ and Gary Lynch ${ }^{2}$ \\ ${ }^{1}$ Center for Neural Science, New York University, New York, New York 10003 and ${ }^{2}$ Center for the Neurobiology of \\ Learning and Memory, University of California, Irvine, California 92717
}

Olfactory memory in control rats and in animals with entorhinal cortex lesions was tested in four paradigms: (1) a known correct odor was present in a group of familiar but nonrewarded odors, (2) six known correct odors were simultaneously present in a maze, (3) correct responses required the learning of associations between odors and objects, and (4) six odors, each associated with a choice between two objects, were presented simultaneously. Control rats had no difficulty with the first problem and avoided repeating selections in the second; this latter behavior resembles that reported for spatial mazes but, in the present experiments, was not dependent upon memory for the configuration of pertinent cues. Control animals varied considerably in their acquisition of odor-object associations with only a subgroup learning every set of pairings. These latter animals also performed well in the fourth task and, as indicated by post hoc analyses, developed complex strategies in dealing with the problem of serial odor-object pairs. Lesioned animals had no difficulty in selecting correct odors learned prior to surgery (problem one) but repeated their choices in problem two. This latter result suggests that hippocampus contributes to the transient memory of prior choices for odors as it does for prior choices in spatial mazes. Entorhinal rats were able to form odor-object associations (problem three), and a subgroup of the animals periodically succeeded in doing a long series of such choices (problem four), though with less frequency than controls. These results indicate that rats use both long-term memory and transient memory in dealing with olfactory problems and suggest that the second of these is dependent upon a hippocampal process that encodes a type of information other than the relationship between cues.

[Key words: olfactory memory, paired-associates, crossmodal learning, olfactory-hippocampal circuit, hippocampus, entorhinal cortex, working memory, cognitive map, spatial memory]

Anatomical and physiological studies indicate that the olfactory system provides substantial and direct input to the hippocampus. The lateral entorhinal cortex, the posterior extension of the olfactory cortex, densely innervates the granule cells of the dentate gyrus and also projects monosynaptically to the hippocam-

Received Apr. 22, 1994; revised July 27, 1994; accepted July 27, 1994.

We thank Gene Go for excellent technical assistance.

Correspondence should be addressed to Dr. Ursula Stäubli, New York University, Center for Neural Science, 4 Washington Place, New York, NY 10003.

Copyright (C 1995 Society for Neuroscience 0270-6474/95/151162-10\$05.00/0 pal pyramidal cells (e.g., Hjorth-Simonsen, 1972; Witter, 1993). Moreover, physiological activity in rat hippocampus becomes synchronized with that in the olfactory bulb and cortex during odor sampling (Macrides et al., 1982). These observations have prompted speculation and experimentation concerning the possible contributions of the several stages of the olfactory-hippocampal circuit to the encoding and use of memory. Lesions to the lateral entorhinal cortex, which separate the hippocampus from its primary source of olfactory input, did not detectably affect the ability of rats to perform odor discriminations learned prior to surgery although they did disrupt the learning of new discriminations (Staubli et al., 1984, 1986). This result suggested that the olfactory cortex and its connections with telencephalic regions other than hippocampus are the sites in which odors are recognized and assigned significance. In accord with this idea, long-term potentiation was found to develop in the piriform cortex when patterned stimulation of the lateral olfactory tract was used as a discriminative cue (Roman et al., 1987).

Hypotheses about which aspects of olfactory memory might be encoded in the hippocampus have, for the most part, been borrowed from studies using nonolfactory cues, for example, the capacity of rats to remember choices made earlier in a spatial maze (Olton et al., 1979), and the increased firing of field CA1 neurons when an improper sequence of visual cues occurs (Ranck, 1973). The extent to which these results hold for odor cues is unknown and indeed there is controversy about their proper interpretation with regard to memory of visual stimuli (see below). Accordingly, the present experiments tested olfactory memory across several behavioral paradigms with the goal of providing information needed for evaluation and further development of hypotheses regarding possible encoding operations by the different stages of the olfactory-hippocampal circuit. The first problem involved long-term memory and required rats to select a known correct cue from a group of simultaneously present familiar odors. Lesions of the lateral entorhinal cortex were used to test if hippocampus contributes to recognition memory in a situation in which the animal is required to reject several possible choices. The second paradigm examined the possibility that rats have a transient or "working" memory which allows them to identify odors responded to earlier in a test session. It has been proposed that memory of this kind, in contrast to longterm recognition/significance memory, is dependent upon hippocampus, and in particular the mossy fiber synapses between the dentate gyrus and field CA3 (Lynch and Staubli, 1991; Lynch et al., 1991; Staubli, 1992). It was also anticipated that the outcome of the experiment would be relevant to a controversy concerning the nature of hippocampal contributions to memory involving spatial cues. Olton and coworkers (1979) showed that 
rats with hippocampal damage make numerous reentries into the arms of a radial maze during a given test session while control animals typically avoid earlier choices. They interpreted this as showing that the hippocampus encodes a transient memory of prior selections. O'Keefe and others (e.g., O'Keefe and Nadel, 1978; Rasmussen et al., 1989) argue that reentry errors in hippocampal animals are secondary to the disruption of a spatial map, that is, a stable memory of the configuration of spatial cues. Tests with odor cues should be useful for determining if memory of prior choices requires hippocampus independent of any role it plays in encoding configurations of cues. The third paradigm involved the learning of associations between odors and objects. Youngentob and colleagues (1990, 1991) have recently shown that rats are adept at associating odors with visual cues in an open field environment. We were interested in testing if animals are able to acquire paired-associates involving odors and intra-maze objects and the extent to which this is dependent upon hippocampus. The hippocampus is the most obvious site for convergence of olfactory and visual information-as noted, it receives a very large input from the posterior extension of olfactory cortex (e.g., Hjorth-Simonsen, 1972; Witter, 1993) and is known to be responsive to visual cues (e.g., O'Keefe and Nadel, 1978; O'Keefe and Conway, 1978). The dense interconnectivity within hippocampus would seemingly provide a substrate for associating contiguous odors and objects. However, O'Keefe and Nadel (1978) have made the important point that hippocampus is not needed for stimulusresponse learning involving intra-maze cues and specific responses, for example, maze learning in the absence of extramaze, spatial cues. From this perspective, it might well be the case that an extrahippocampal system encodes and uses intra-maze associations of odors with objects. The fourth paradigm combined problems two and three: rats were required to make a series of odor choices each of which was followed by an object choice. This provided (1) a test of the rat's ability to integrate transient memory (which odors were already selected) and longterm memory (which object is associated with the odor just selected), and (2) an opportunity to determine if any deficits in transient memory of choices caused by hippocampal denervation would impair utilization of long-term memory.

\section{Materials and Methods}

Subjects. Ten male Sprague-Dawley rats, 3 months of age at the beginning of the study, were used. They were housed individually and trained and tested during the dark phase of their $12 \mathrm{hr}: 12 \mathrm{hr}$ light-dark cycle. Access to food was restricted to maintain the animals at $90 \%$ of control body weight, and water was available ad libitum. The same 10 animals were run in each of four experiments during a period of over 12 months.

Behavioral apparatus. The apparatus consisted of a modified elevated radial maze made of painted wood that was kept in a semidark room with minimal lighting. The center platform was $35 \mathrm{~cm}$ in diameter with 12 radial arms that were $90 \mathrm{~cm}$ long, $7 \mathrm{~cm}$ wide at the entrance and extended progressively to a width of $25 \mathrm{~cm}$ at the distal end. Each arm was fully cnclosed by $25 \mathrm{~cm}$ high walls except at the entrance where a $5 \mathrm{~cm}$ high wooden hurdle was located which the rat had to climb over to get access from the center platform. A hole drilled in the floor at the distal end of each arm served as hidden food well. Odors were prepared by enclosing a small amount $(\sim 3 \mathrm{ml})$ of a given odor concentrate (International Flavors and Fragrances, Inc.) into a $50 \mathrm{ml}$ Erlenmeyer flask and gassing it with air monitored by a flow-meter at a rate of $0.1-1.0$ liters/min (depending on the intensity of the odor concentrate used). The odor-saturated air leaving the flask then joined with a clean air stream regulated by a second flow-meter at $4.5-5.5$ liters $/ \mathrm{min}$, such that the final volume combined of clean and odor-saturated air delivered per minute was identical for each odor. The odorized air was carried via Tygon tubing to the maze and ejected towards the center platform
A

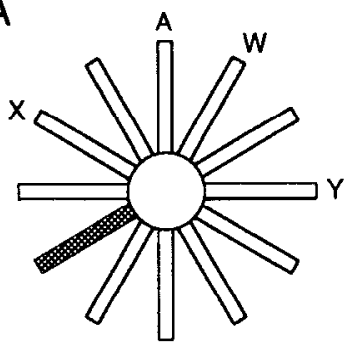

Z

C

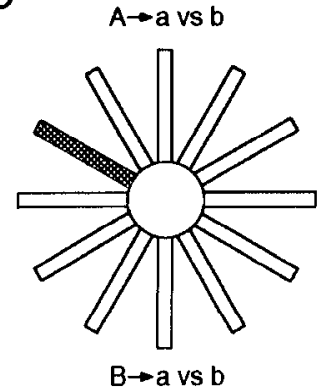

B

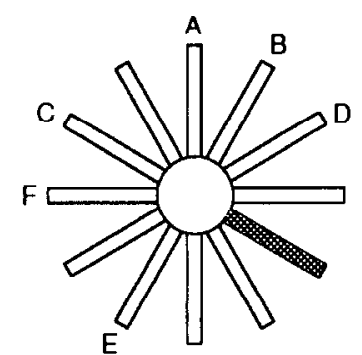

D

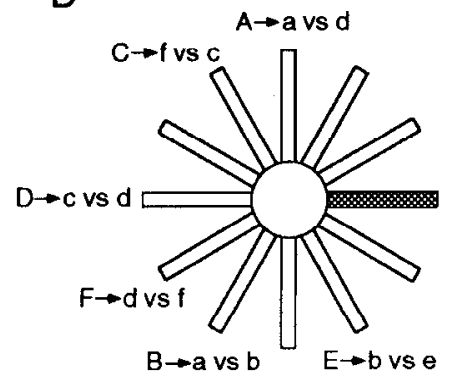

Figure 1. Illustration of the various paradigms used in the study. Shape and proportions of the 12 arm maze are schematized and do not exactly correspond to the actual maze (dark arm $=$ start alley). $A$, Long-term recognition memory: choose correct odor $(A)$ from a group of five familiar odors; $B$, olfactory working memory: sample each of six odors only once within a trial, irrespective of odor rotations and delays interposed after the third choice; $C$, Cross-modal paired-associates between odors and objects: associatc odor $A$ with object a and odor B with object $b$ (phase 1), then odor $C$ with object $c$ and odor $D$ with object d (phase 2), and last odor $E$ with object $e$ and odor $F$ with object $\mathrm{f}$ (phase 3); $D$, serial odor-object associations: solve all six odor-object associations within a single trial.

through an opening in the hurdle located at the entrance to each alley. A fan mounted $25 \mathrm{~cm}$ above the center platform served to exhaust the odors.

Experiment 1: long-term memory for significance of simultaneously present odors. Rats were trained to discriminate between five odors ejected simultaneously from different arms of the radial maze (see Fig. 1A). Two sets of four odors were used; the members of the first set [celery $(\mathrm{X})$, rose $(\mathrm{Y})$, garlic $(\mathrm{Z})$, basil $(\mathrm{W})$ ] werc present in cach trial and always marked arms with empty food wells; the fifth odor was randomly selected in each trial from the second odor set [coconut (A), banana (B), chocolate (C) and cheese (D)] and always denoted an arm containing a food reward (cheese bits, banana chips, coconut chips, and chocolate chips, respectively). The five odors were assigned to five different arms in every trial and one of the arms containing no odor served as start alley. The two arms directly adjacent to the right and left of the start alley were never used. Animals were allowed to make one choice per trial and were then removed to their home cage for 10-15 min; five trials were given per day. Training was conducted for several weeks until each animal made three or more correct choices per day across 5 consecutive days. Five of the animals were randomly picked and subjected to bilateral electrolytic lesions of the entorhinal cortex. Testing was resumed one week later during $10 \mathrm{~d}$, using identical procedures and odors as preoperatively.

Memory for odors sampled during a testing session: "working memory" task. Six odors were used that were released simultaneously from randomly selected and baited arms of the radial maze as shown in the illustration of Figure $1 B$. The odors included the set of four odors marking food rewards in the first experiment plus two additional odors, that is, butter odor (E) with Fruit Loop bits as reward, and walnut odor (F) with walnut bits as reward. Correct performance consisted in responding to each odor once and collecting the food. Rats received one trial per day for several weeks. The actual experiment involved $15 \mathrm{~d}$ of testing with no delay, followed by 15 trials with a delay after the third choice during which the rat was confined inside a large nontransparent 


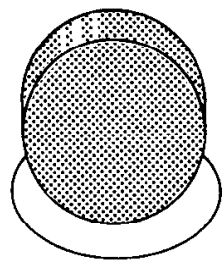

Banana

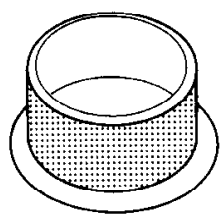

Cheese

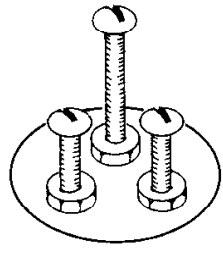

Butter

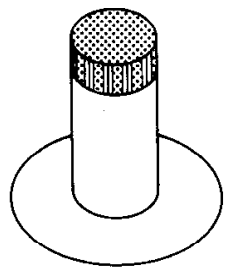

Chocolate
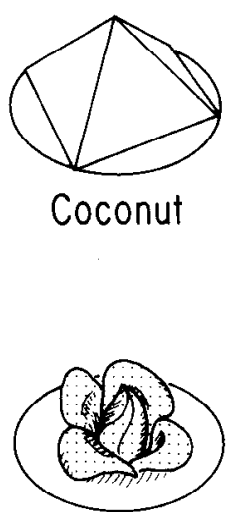

Walnut
Figure 2. Illustration of the six three-dimensional objects, each of which was paired with the odor listed underneath. The objects were glued onto a circular cardboard of $3.5 \mathrm{~cm}$ in diameter, large enough to cover a food well.

cylinder in the center area and the maze was either rotated by $90^{\circ}$ or all six odors were reassigned to different arms. These control procedures interposed a delay of approximately 3-5 min between the first and second set of three choices.

Cross-modal associations: "paired-associates" between odors and objects. Each odor used in the preceding experiment was paired with one of six three-dimensional object as illustrated in Figure 2 . An insert with two recessed food wells was placed at the end of all arms. Training was conducted in three phases that each involved two different odor-object pairs and lasted several weeks. To obtain a reward, the animal had to enter both odor-releasing arms and select the correct of two objects. A food reward was hidden underneath the correct object, removing the wrong object uncovered an empty well. The animals encountered the same object pair in both arms such that the correct object in one arm was incorrect in the second arm, and vice versa (for an illustration of the experimental design, see Fig. $1 C$ ). Left-right positions of baited food wells varied randomly within and across five daily trials. Animals were removed to their home cage for 15-25 min between trials. A strong spot light positioned on the ceiling above the center of the maze served to enhance visibility of the objects. Training on each set of odor-object pairs was carried out for several weeks. To test if object choice was guided by food smells, probe trials were carried out with no food present (the animal received the reward from the experimenter upon displacing the correct object) or with food under the wrong object.

Serial odor-object associations. The serial version of the paired-associate task required that all six odor-object pairs introduced in the preceding experiment were solved sequentially within a single trial. Thus, all six odors were released simultaneously and each odor led to two objects (see Fig. 1D). In contrast to the preceding experiment the incorrect object placed with a particular odor varied from trial to trial. Individual odors were released from different arms in each trial. A trial was terminated upon displacement of a wrong object, while repeated entries into odor arms were noted but did not result in termination of a trial. Each animal received five daily trials for $18 \mathrm{~d}$ with the animal in the home cage for 15-25 min between trials.

Lesions. Bilateral entorhinal cortex lesions wcre performcd since (1) our previous studies on the hippocampal role in olfactory memory used entorhinal lesions to disconnect the hippocampus from its olfactory input (Staubli et al., 1984, 1986) and (2) studies by others on spatial memory have indicated that damage to the entorhinal cortex results in deficit patterns similar to those found after extensive damage to the hippocampus proper (Jarrard et al., 1984; Schenk and Morris, 1985; Rasmussen et al., 1989). Animals were pretreated with atropine $(0.1$ $\mathrm{mg}$ ) to reduce salivation and anesthetized with a mixture of ketamine $(50 \mathrm{mg} / \mathrm{kg})$ and Rompun $(10 \mathrm{mg} / \mathrm{kg})$ injected intraperitoneally. Bilateral electrolytic ablations of the entorhinal cortex were conducted identical to protocols described earlier (Staubli et al., 1984, 1986). Stereotaxic coordinates in reference to lambda were posterior -0.8 , lateral 3.0 ,

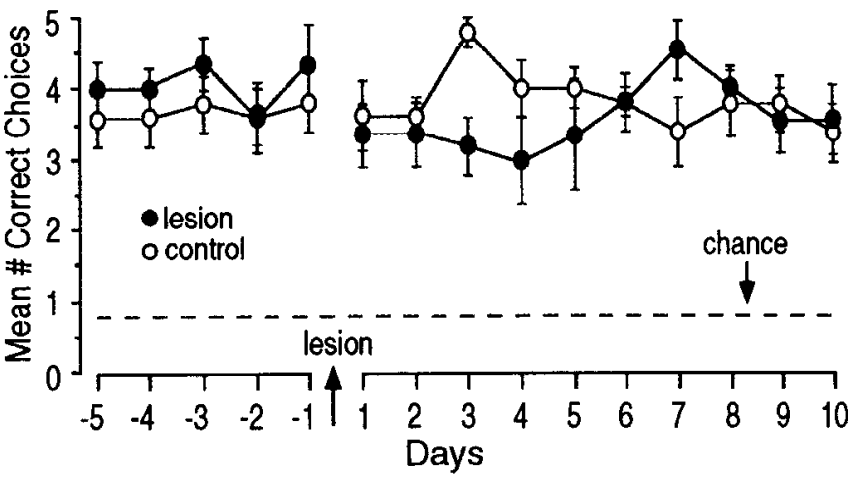

Figure 3. Effects of bilateral entorhinal cortex lesions on recognition memory, that is, the ability to remember and distinguish the correct one out of five familiar odors presented simultaneously. The task involved a total of four correct and four incorrect odors, with the same four incorrect odors presented in every trial while the fifth and correct odor varied randomly across five daily trials. Performance is shown for the last 5 preoperative days and for $10 \mathrm{~d}$ following recovery from the lesion.

ventral $-6.0,-4.0,-2.0$; posterior -0.8 , lateral 4.0 , ventral -7.0 , $-5.0,-3.0$; posterior -0.8 , lateral 5.0 , ventral $-7.0,-5.0,-3.0$; all at an angle of $10^{\circ}$ from the vertical midline and with the toothbar set at -5.0 . Current of $1 \mathrm{~mA}$ was passed for $45 \mathrm{sec}$ at each of the above described nine placements using an insulated stainless steel electrode that was cut at the tip. The five control animals were prepared identically except that no current was passed (sham lesions).

Histological processing. After completion of testing all animals were deeply anesthetized and perfused transcardially with buffered saline solution followed by $10 \%$ buffered formaldehyde solution. The brains were postfixed in formaldehyde solution for $24 \mathrm{hr}$ and sunk in 10\% buffered sucrose solution overnight. They were then frozen and cut coronally at $40 \mu \mathrm{m}$ starting at the septal end of the hippocampus. Horizontal sections were taken from the last third of hippocampus and adjacent entorhinal cortex. Every fourth coronal section was stained for acetylcholinesterase, a histochemical procedure which allows determination of the extent of denervation in hippocampus produced by damage to the retrohippocampal area (Lynch et al., 1972; Cotman et al., 1973). Every fourth horizontal section was stained with cresyl violet to verify the extent of actual tissue loss in the area of lesion. All sections were mounted on microscope slides and examined with a desk projector.

\section{Results}

Long-term memory for significance of multiple, simultaneously present odors

Olton and colleagues (1979) trained rats in a radial maze in which a subset of arms (spatial locations) was always rewarded and a second set was not, and found that hippocampal lesions did not cause the animals to confuse the two groups of arms (i.e., the experimental animals entered correct locations and avoided incorrect arms). These authors considered the longterm memory of correct versus incorrect positions in the maze as a kind of "reference" memory and concluded from their results that it is not dependent upon hippocampus. The first study tested if these observations hold true for olfactory memory, that is, are rats able to distinguish the significance of several simultaneously present odors and do they retain this ability after lesions to the primary olfactory input to hippocampus? Several weeks were required to train the animals on this task, which involved different phases of learning, such as acclimation to the testing situation, learning that odors are the relevant cues (i.e., ignoring spatial and nonolfactory intramaze cues) and then mastering a few simple "rules" (leave the current arm, enter the decision point, follow an odor, find the reward, etc.). As illustrated in Figure 3, during the last $5 \mathrm{~d}$ before surgery both the 
intact and control groups were equally successful in picking correct odors. In the first five postoperative testing days experimental animals confused the two subsets of odors more frequently than controls $[t(8)=3.09, p<0.015$, two-tailed $t$ test, days $1-5$ postsurgery] as well as compared to their own preoperative performance $[t(4)=6.32 p<0.003$, two-tailed paired $t$ test, days $1-5$ pre- vs postsurgery]. This deficit was temporary as evidenced by a recovery of their scores during days 6-10 to levels indistinguishable from preoperative performance $[t(4)=$ $0.69, p<0.52$, days $1-5$ pre vs $6-10$ post]

\section{Memory for odors sampled during a testing session: "working memory"}

Rats in radial mazes quickly learn to avoid arms from which they have already removed the reward. Their memory in this regard is impressive since the animals enter and subsequently avoid reentering many arms even when a delay of several hours is interposed in the middle of the testing session. Olton and coworkers (1979) suggest that this type of memory is analogous to "working memory" (in essence a list of itcms tested during a several hour period) and argue that its disruption is at least in part responsible for the profound impairment in spatial mazes produced by hippocampal lesions. A counterhypothesis (e.g., O'Keefe and Nadel, 1978; Rasmussen et al., 1989) is that the hippocampus encodes spatial relationships using extramaze visual cues and that lesions to the structure disrupt the utilization of such information thereby producing random behavior in the maze (i.e., numerous reentries into already sampled arms). In this view, the effects of hippocampal lesions on what Olton and colleagues (1979) refer to as working memory are secondary to a disturbance of memory involving spatial relationships. In the present experiment we asked (1) if rats possess a working memory for odors in a situation in which spatial relationships are irrelevant to performance and (2) if such a memory is disrupted by disconnecting the olfactory efferents to hippocampus.

Overall, the animals were quick at learning this task. $\Lambda$ s illustrated in Figure $4 A$, they randomly picked any of the six odors as their first choice in a trial (chance level, 16.7\%), with the exception of cheese odor (D) which was selected in approximately a third of total trials (31\%), significantly more often than butter- (E) $[t(8)=3.28 ; p<0.004]$, banana- $(\mathrm{B})[t(8)=2.83$; $p<0.01]$, and chocolate-odor (C) $[t(8)=2.68 ; p<0.02$; twotailed $t$ test]. Obviously, the animals' choices were guided by olfactory rather than spatial cues. The intact group committed approximately one reentry error in trials that did not involve. maze rotation or odor reassignment after the third odor choice (Fig. 4B). Animals with lesions to the entorhinal cortex searched and sampled the different odors and consumed rewards but made significantly more reentries than controls $[t(8)=3.92, p$ $<0.004$; control vs experimental group; two-tailed $t$ test; Fig. $4 B$ ). Intact rats succeeded in sampling all odors without reentries in more than a third of total trials (Fig. $4 C$ ); their number of error-free runs (" $x$ " in Fig. 4) decreased in trials with a delay interposed, consistent with findings of a time-dependent working memory decay during delayed olfactory match-to-sample performance (Otto and Eichenbaum, 1992; Staubli et al., in press). Control animals made the majority of first errors on their sixth choice, that is, after five correct entries, both in trials with or without a delay. In contrast, the distribution of scorcs from lesioned rats was not statistically different than expected from random performance [Kolmogorov-Smirnov test of goodness of fit in lesioned rats: $D=0.13$ (no delay), $D=0.20$ (delay); $p$
A

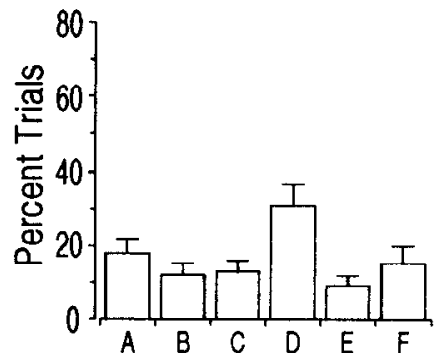

B

C
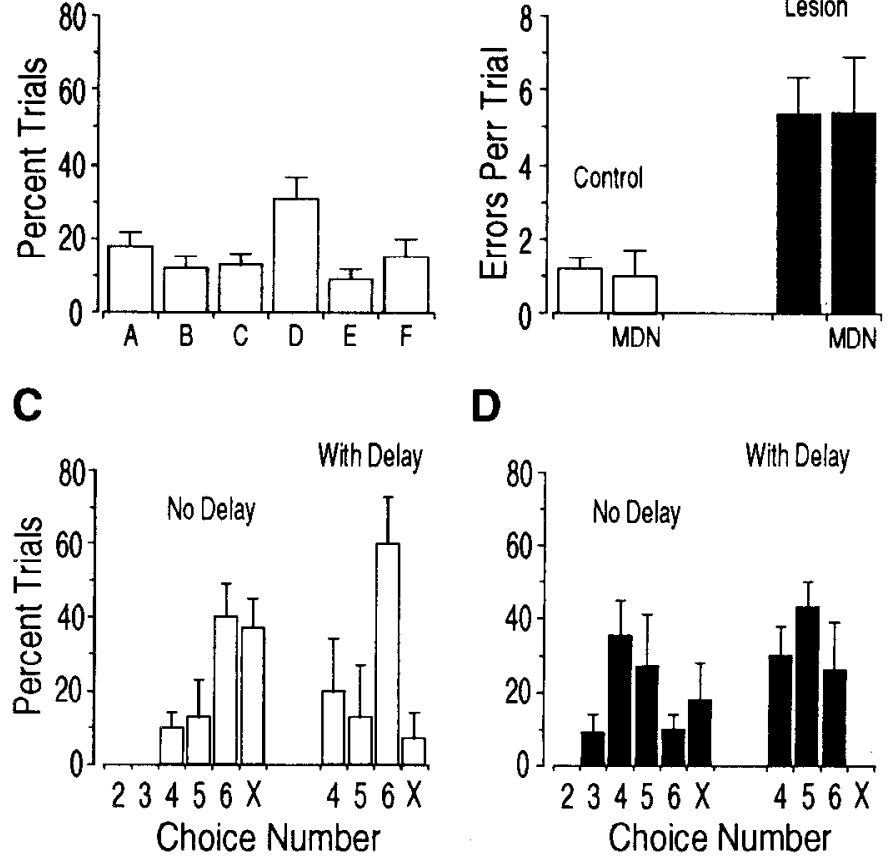

D

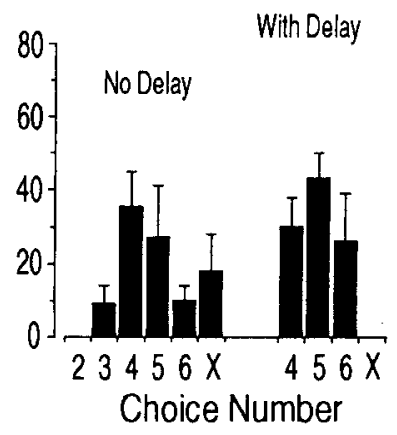

Figure 4. Effects of bilateral entorhinal cortex lesions on memory for odors already responded to earlier in the test session, that is, olfactory working memory. $A$, Percentage of total trials in which individual odors were selected as first choice by the 10 animals ( $A$, coconut; $B$, banana; $C$, chocolate; $D$, cheese; $E$, butter; $F$, walnut). $B$, Mean and median $(M D N)$ number of reentry errors committed by the control and experimental group in 15 trials without delay. $C$, Choice on which control animals made their first reentry errors in trials in which a delay was or was not interposed after the third odor selection. $X$ denotes the percentage of trials in which no reentries were made. $D$ same as in $C$ but for the experimental group.

$>0.20$; for control rats: $D=0.57$ (no delay), $D=0.47$ (delay); $p<0.01$ ]; however, the entorhinal animals did not perseverate as they did not repeat their initial choices with a frequency greater than that expected from chance. These observations provide the first evidence that rats are adept at maintaining a list of odors in memory and that this capacity is substantially reduced by severing the olfactory projections into hippocampus.

\section{Paired-associations between odors and objects}

While rats rapidly learn to use spatial cues to locate rewards, they are surprisingly slow to form associations between a specific extramaze cue and reward (O'Keefe and Nadel, 1978; Rasmussen et al., 1989). This suggests that different types of learning are involved in the two tasks, and indeed, hippocampal lesions that disrupt performance in spatial mazes do not do so for problems involving simple visual associations ( $\mathrm{O}$ 'Keefe and $\mathrm{Na}$ del, 1978; Schenk and Morris, 1985). The present experiment tested if rats are able to learn associations between odors and objects and if denervation of the hippocampus interferes with the ability to form such memories. The hypothesis was that associations between odors and objects are mediated by hippocampal circuits.

It was apparent that a subset of animals in each group mastered odor-object associations more easily than others. The animals required $20 \mathrm{~d}$ of training to learn the first set of pairings (at least $80 \%$ correct across $5 \mathrm{~d}$ ) and $30 \mathrm{~d}$ for the second and 

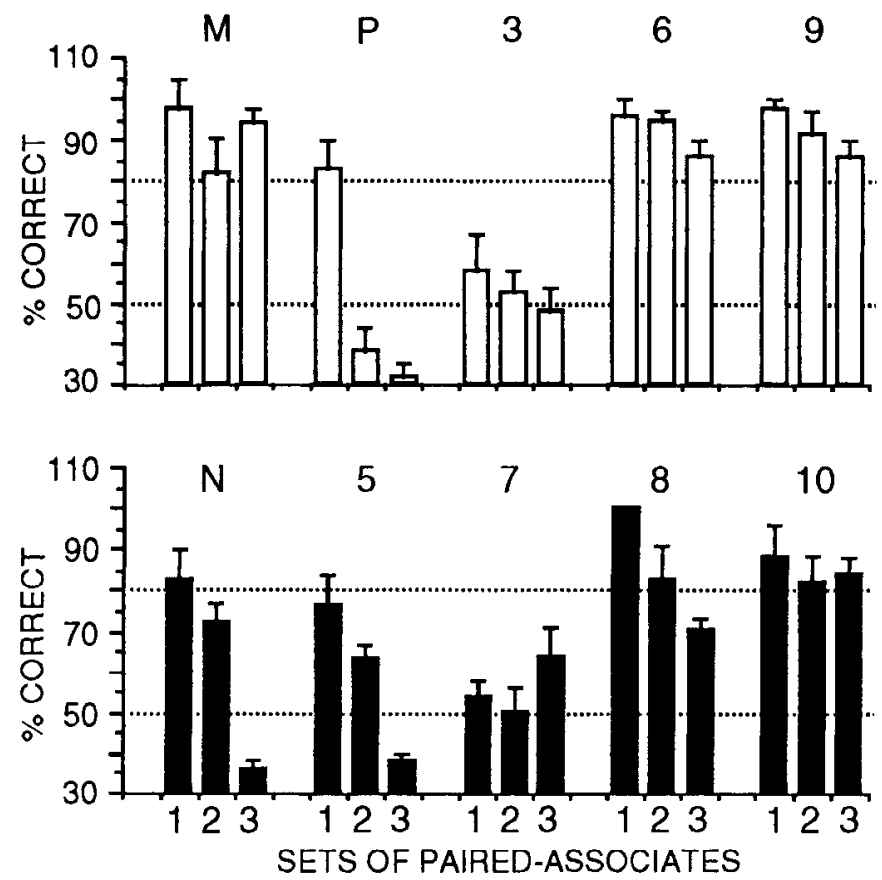

Figure 5. Acquisition of odor-object associations (paired-associate learning). Five control animals (open bars) and five animals with bilateral entorhinal cortex lesions (solid bars) were tested for their ability to associate six familiar odors with a specific three-dimensional object. Training was conducted in three phases $(1,2,3)$ entailing each a set of paired-associates (two different odor-object pairs). Bars denote the amount of correct odor selections made in 25 trials during the last $5 \mathrm{~d}$ of a training phase. Stippled horizontal lines correspond to chance performance (50\%) and learning criterion (80\%). Letters above bars correspond to individual rats.

third sets. Figure 5 illustrates performance during the last $5 \mathrm{~d}$ of training on each set. Three of the animals in the control group learned all odor-object associations ( $>80 \%$ correct), a fourth animal acquired the first set but failed on the second and third set, while the fifth animal never surpassed chance level $(50 \%)$. In the entorhinal group, one animal acquired all three sets, a second animal mastered the first two sets, a third animal learned the first set well ( $>80 \%$ correct) and the second one reasonably well $(72 \%)$, and a fourth animal only acquired the first set. In all, the scores of the two most deficient animals in the entorhinal group $(5,7)$ and the control group $(\mathrm{P}, 3)$ were comparable; similarly, the best learners in the entorhinal group $(8,10)$ were not obviously different from the three animals with highest scores in the control group $(\mathrm{M}, 6,9)$.

Probe trials involving a single odor released from two locations indicated that the high scores of the above subset of learncrs were not duc to object altcrnation (i.c., if object \#1 is rcwarded to first odor, choose object \#2 in second arm). Similarly, tests in which (1) both objects in a given arm covered empty wells or (2) food was placed under the incorrect object did not interfere with the superior performance of these animals.

\section{Performance in a maze requiring serial odor-object associations}

This study measured the effect of hippocampal denervation on the utilization of long-term memory in circumstances that require the rat to maintain in parallel a particular mode of searching, cue approach and sequential decision making. In essence, this paradigm combined experiments 2 and 3 , and thus required
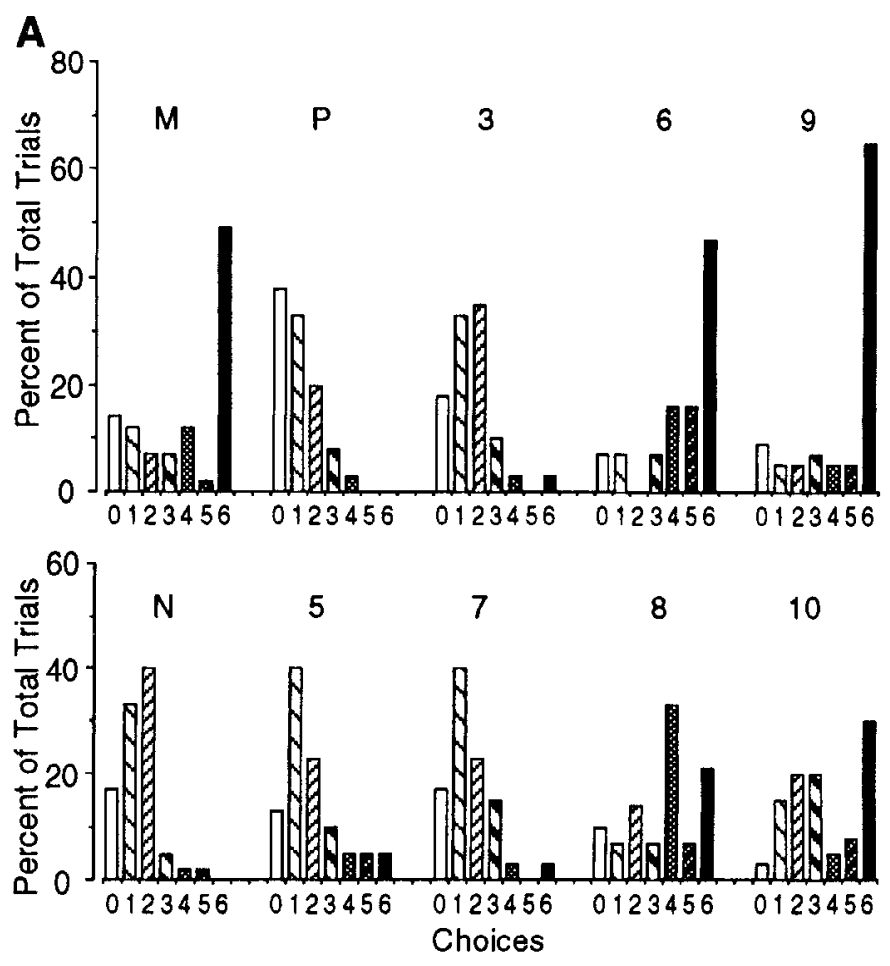

B

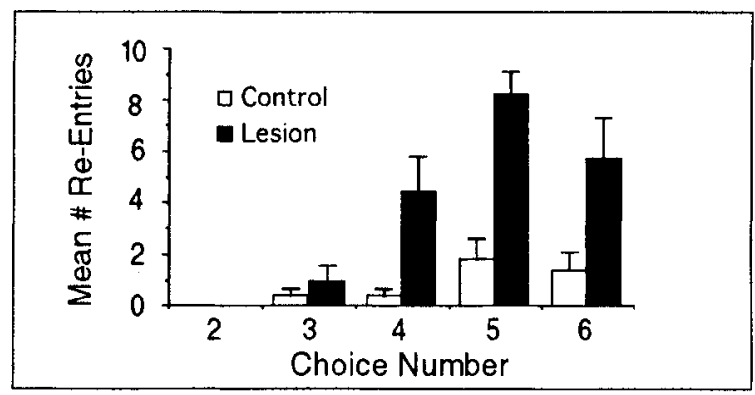

Figure 6. Serial odor-object associations involving six simultaneously present odor-object pairs. $A$, Correct choices within a trial. Percent trials in which intact animals $(t o p)$ and animals with bilateral entorhinal cortex lesions (bottom) made from $0-6$ correct choices. Displacing the incorrect object resulted in termination of the trial. The results shown were collected over $15 \mathrm{~d}$ of five daily trials. $B$, Working memory errors. Mean number of repeated visits to the same odors by entorhinal and control animals in trials with $2-6$ correct choices. The experimental group made significantly more errors than controls $(p<0.02, p<0.0007, p<0.04$ for choice number 4,5 , and 6 , respectively; two-tailed $t$ test).

the rat to sample each of six odors within the same trial and to select the correct object associated with each specific odor. Working memory components of the task were minimized by not penalizing the rats for approaching already selected odors. Correct performance thus entailed moving through the maze using cues (odors) that had no explicit relationships with each other and making a series of decisions based on associations in long-term (reference) memory.

The three intact animals $(M, 6,9)$ that had learned all odorobject relationships in the preceding experiment performed quite well on this paradigm; that is, they accomplished error-free runs in $49 \%, 47 \%$, and $65 \%$ of total trials, respectively (Fig. 6A). Similarly, the two entorhinal rats that had performed well in the previous study $(8,10)$ made six correct choices in $21 \%$ and $30 \%$ of total trials, respectively (chance, $2 \%$ ). The remaining 

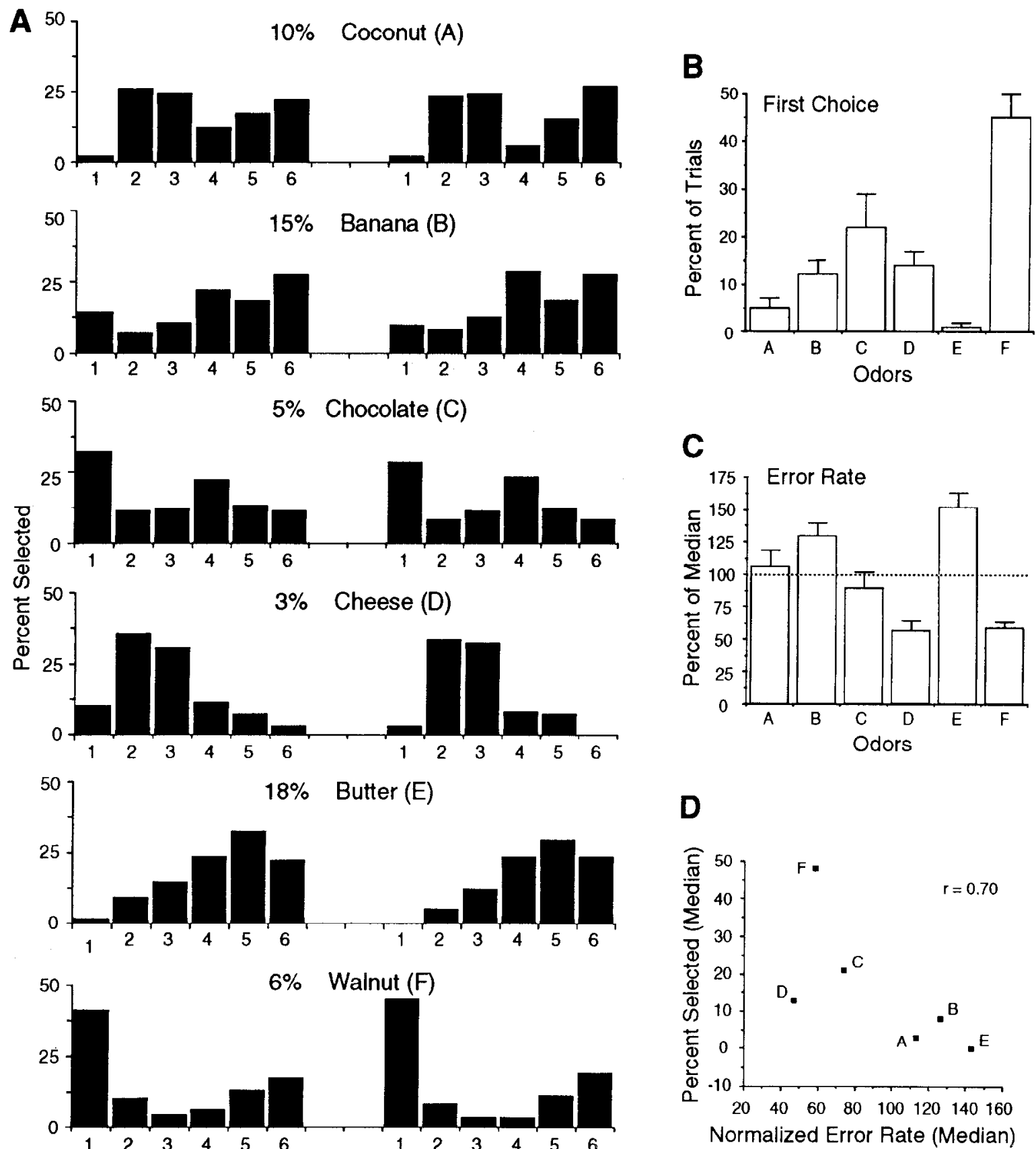

Figure 7. Relationship between type of odor selection, time point of selection (choice number), frequency of selection and error rate for three control rats $(M, 6,9)$ during performance of the serial odor-object association task. $A$, Frequency at which each odor was selected as first, second, third, fourth, fifth, or last choice in a trial. Data are expressed relative to total number of choices counted for each odor. Mean values are depicted on the left and median values on the right half, and the number above each subgraph indicates the fraction of total responses made to a particular odor that were incorrect (absolute error rate). $B$, Percentage of total trials at which each odor was first choice. $C$, Normalized error rate; that is, percentage incorrect responses to each odor expressed relative to the median number of incorrect responses for all odors. $D$, Relationship between first choice selection and relative (normalized) error rate, that is, success rate.

animals in both groups were impaired and finished the majority of trials with three or fewer correct sequences. In cases with four or more correct choices, entorhinal rats committed significantly more reentry errors (working memory errors) than controls (see Fig. $6 B$ for significance levels). It is noteworthy, however, that the lesioned group made substantially more correct first choice selections than expected from random performance $(50 \%)$ and did not differ from the control group (percentage of trials with correct first choices: control $82 \pm 5 \%$ vs lesion $83 \pm 4 \%$, mean \pm SEM)

Performance of the three control animals $(M, 6,9)$ that came closest to mastering the serial odor-object problems was analyzed in more detail (Fig. 7). Three of the odor-object relationships were easier for these animals than the remaining three. Thus, incorrect choices were made on $3 \%, 5 \%$, and $6 \%$ of the trials for three odors (D,C,F) and $10 \%, 15 \%$, and $18 \%$ of the 


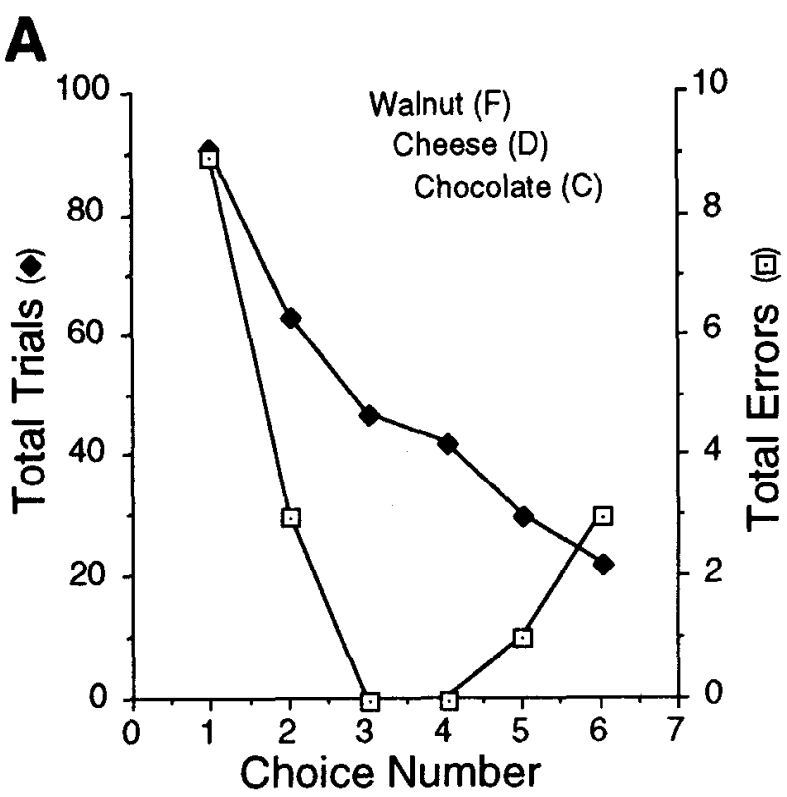

B

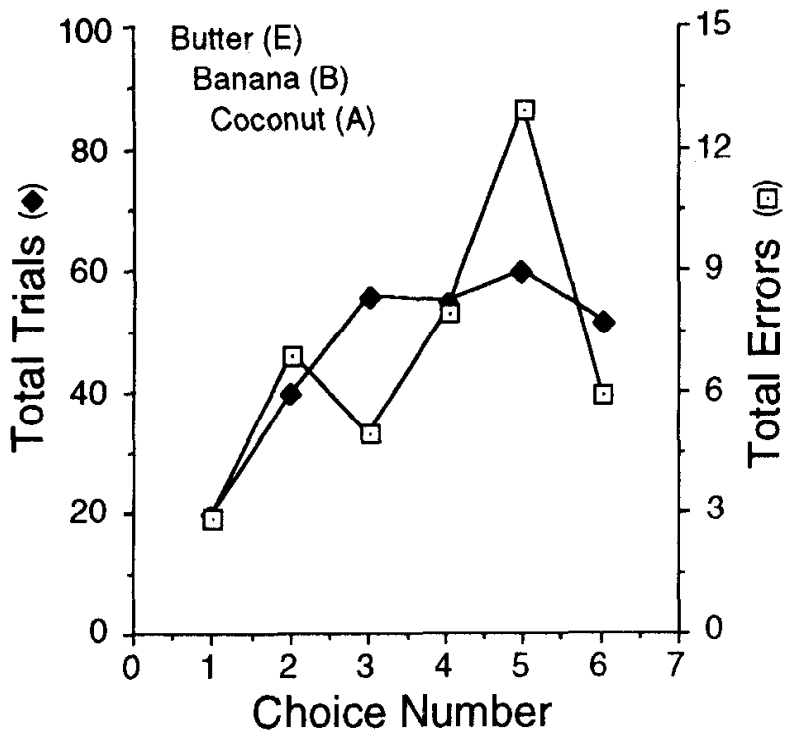

C

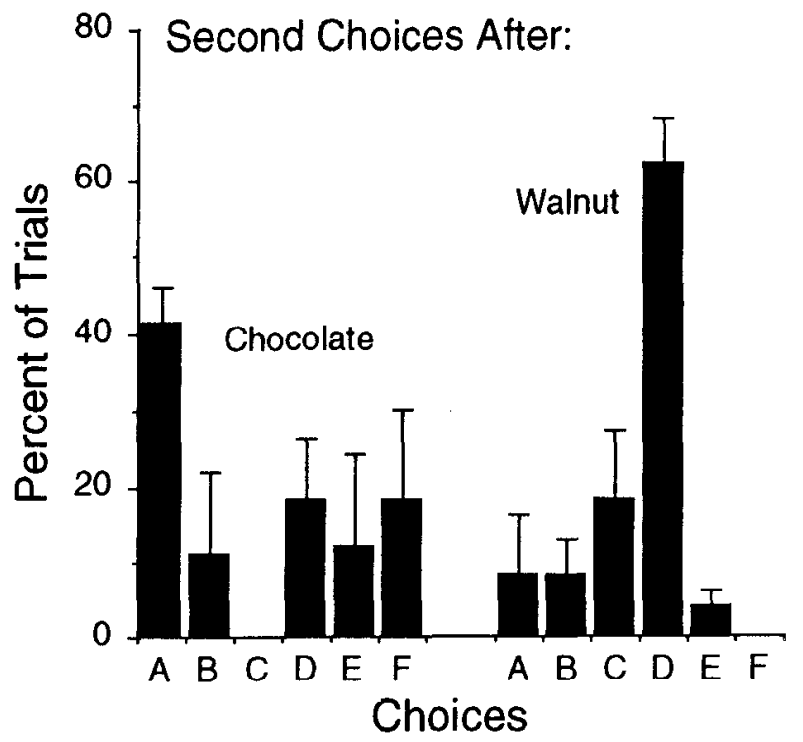

trials for the remaining group (A,B,E) (see Fig. 7A). Interestingly enough, the rats generally selected one of the odors with a low error rate (walnut, chocolate, cheese) for their first or second choice and tended to avoid those with higher error rates (butter, banana, coconut) until late in the trial. Comparing first choice selections between odors across all trials confirmed that the three odors with a low relative error rate were selected more often as first choice than the remaining three odors (Fig. $7 B, C$ ). Indeed, the probability that an odor was selected as a first choice was correlated with the probability of success with that odor $(r=$ 0.70 ; see Fig. $7 D$ ); this correlation was significant when evaluated by Spearman's rank order correlation $\left(r_{s}=0.82, p<0.05\right)$. The number of occurrences for odor selcctions with low error rate (odors $\mathrm{F}, \mathrm{D}, \mathrm{C}$ ) decreased linearly across successive choice numbers (Fig. $8 A$ ) and increased for odors with high error rate (Fig. $8 B$ ), and in both cases the number of errors per selection had no obvious relationship to choice number; that is, error rate for a given odor was independent of when, in a series of choices, it was selected. Interestingly, the first odor selected in a trial biased subsequent odor choices, that is, the animals made different second choices depending on whether walnut- or chocolate-odor was the first choice (walnut- and chocolate-odor were first choices in $2 / 3$ of total trials). Specifically, walnut-odor clearly predisposed the animals to choose cheese-odor (D) as second choice over any other odor $(T=3.75 ; p<0.0003)$, while chocolate-odor as first choice did not cause a significant trend for a specific second choice (see Fig. $8 \mathrm{C}$ ). Curiously enough, the two odors that were preferred as first choice (walnut- and chocolateodor) were not favored as second choice.

\section{Histological analyses}

Examination of horizontal sections revealed that all lesions bilaterally included the lateral entorhinal cortex, the projection zone of the lateral olfactory tract. In all cases the damage also extended into medial entorhinal cortex and caudal hippocampus and included part of the subicular complex. Examination of coronal sections revealed a dense band of acetylcholinesterase in the entorhinal projection area of the molecular layer and confirmed the massive deafferentation expected from this pattern of damage (Fig. 9). No correlation was apparent between the extent of the lesion in individual animals and the degree of impairment in any of the behavioral measures.

\section{Discussion}

The present results confirm and extend previous findings showing that rats have stable memories for odors (Staubli et al., 1986, 1987, 1989). The task used in experiment one was somewhat more difficult than the two-odor discrimination paradigm employed in the earlier work. The rat was confronted with five choices with the correct cue varying across days; the greater difficulty of the task is evidenced in the higher error rates than

\footnotetext{
Figure 8. Lack of relationship between numbers of errors per selection and time point of selection. Bias for second odor choice as a function of first odor selection. $A$, Frequency of selection, choice number and frequency of errors observed for subgroup of three odors with high success rat (low error rate); data are from the same group of three control rats $(\mathrm{M}, 6,9)$ shown in Figure $7 . B$, Same as in $A$, but for the subgroup of three odors with high error rate. $C$, Three control rats $(M, 6,9)$ make different second choices depending on whether walnut or chocolate odor was first choice.
} 

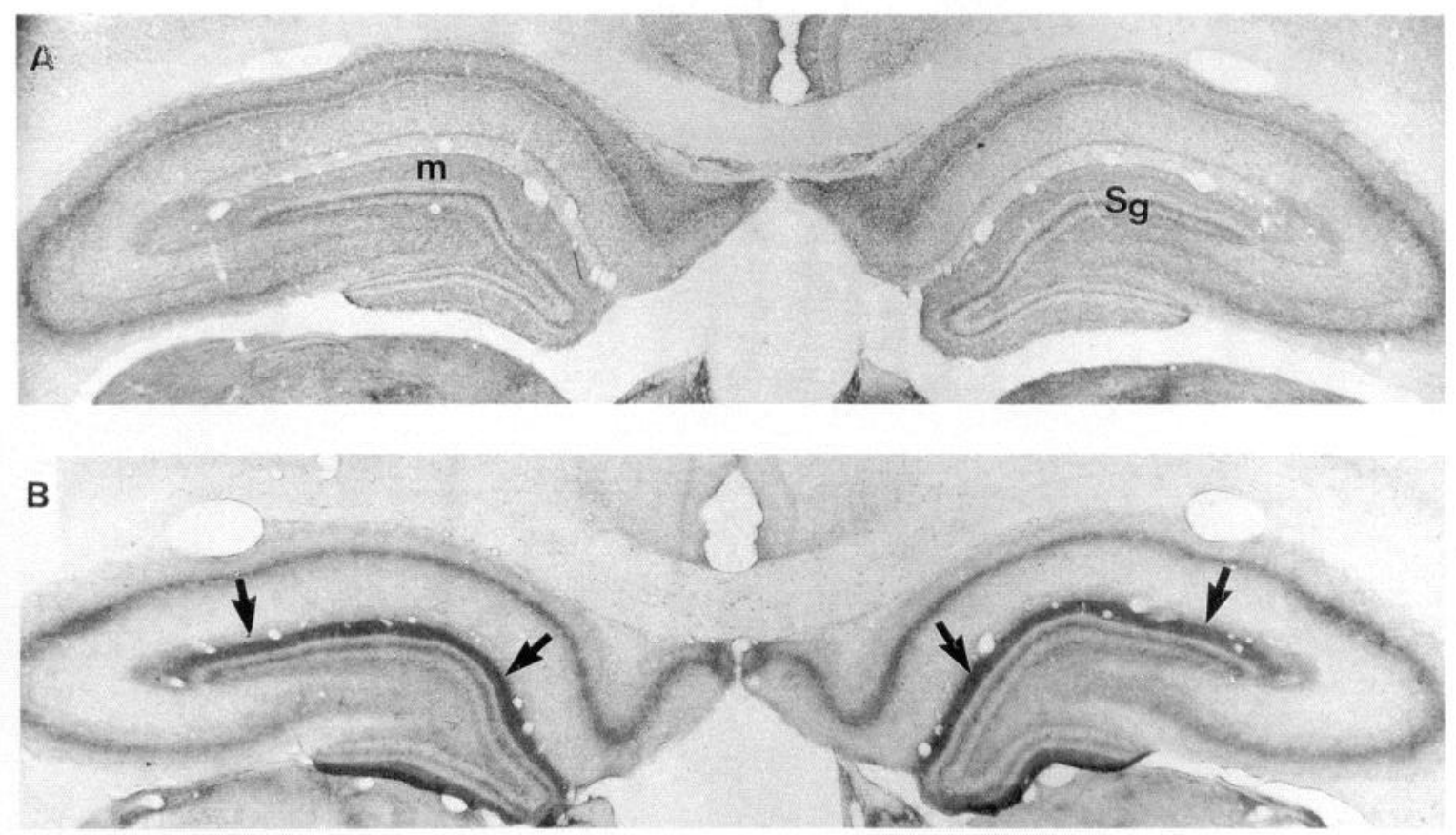

Figure 9. Coronal sections of a sham-lesioned $(A)$ and entorhinal-lesioned $(B)$ brain stained for AChE activity. The entorhinal cortex lesion caused significant shrinking of the outer two thirds of the molecular layer $(m)$, that is, the dendritic zone of stratum granulosum $(S g)$ which is normally innervated by entorhinal cortex afferents. The wide band of dark stain in the shrunken area (indicated by arrows) reflects lesion-induced sprouting of AChE-containing terminals into the deafferented zone.

occurred in two-odor discrimination learning. Lesions cutting the primary olfactory input to hippocampus did not eliminate retention or utilization of the long-term memories used in the task. These results indicate that recognition memories as well as the systems needed for sampling across odors, withholding responses, etc., do not depend upon the flow of olfactory information through hippocampus. Physiological studies using slices and chronic animals have now established that the primary olfactory cortex has LTP-like plasticity (Jung et al., 1990; Kanter and Haberly, 1990) and that patterned stimulation of the lateral olfactory tract used as a discriminative cue produces both learning and LTP (Roman et al., 1987); it is thus reasonable to assume that the cortex itself provides a site for encoding of recognition memories. The extensive projections from the periamygdaloid subdivision of olfactory cortex into the amygdala (Ottersen, 1982) could then be used by the brain to evaluate the value of the cue with amygdala-striatal connections (Groenewegen et al., 1980; Kelley et al., 1982) providing for appropriate responses (approach, ignore).

Rats were found in the second study to avoid reentering arms containing odors which had already been responded to earlier in the session; the imposition of a delay and a rearrangement of odor positions caused only a slight disturbance of this behavior. It seems then that the animals have a memory system for recent cue-response sequences. It was apparent that longterm memory of the configuration of the odors was not involved in this type of memory since the positions of the odors varied across days-presumably the animals encoded each of the six odors and their responses to them into transient memory. The olfactory memory involved in avoiding repetitive selections may be analogous to the transient (4-8 $\mathrm{hr})$ memory used in spatial mazes where extramaze visual cues guide behavior. In the latter case, the memory decays significantly after $6 \mathrm{hr}$ (Gallagher et al., 1983; Staubli et al., 1994), an observation that could be useful for testing the relationship of the memory systems employed in the spatial versus olfactory paradigm. Denervation of the hippocampus caused a substantial impairment of performance in the olfactory maze. This could not be ascribed to perseveration since the animals began to make reentry errors only later in the test session, that is, only after there was a good statistical chance for a repeated choice assuming responses were made randomly. Experiment one indicated that rats with denervated hippocampi had no difficulty in discriminating between the odors and in withholding incorrect responses. A major difference between experiment one and two was that incorrect odors were encoded into long-term memory in the first study and into transient memory in the second study, that is, an odor was incorrect because of recent (positive) experience with it. It is thus not unreasonable to assume with Olton and colleagues (Olton et al., 1979; Olton and Feustle, 1981; Raffaele and Olton, 1988) that the hippocampus is the site of transient encoding for recent decisions (cue-response sequences) and that this function (i.e., working memory) is independent of the contributions of hippocampus to long-term memory of spatial relationships.

The third experiment demonstrated that rats are capable of acquiring cross-modal paired-associates between odors and objects. The first set of pairings was acquired relatively quickly while the remaining two proved to be more difficult; indeed, two of the control rats exhibited poor performance even with extensive training. This variation across problems may reflect differences in the difficulty of the odor-odor or object-object discriminations. Interestingly, the entorhinal group showed a very similar pattern of acquisition in that the second and third sets of odor-object associations proved to be more difficult to master (see Fig. 5). Two animals with lesions performed well in the task while a third and fourth at least partially mastered the first two sets; the remaining subject showed little signs of learning. These differences did not correlate with performance 
in the "working memory" problems; rats with pronounced deficits on the earlier task did not appear to be particularly impaired in the odor-object association paradigm. If the hippocampus is not needed for this type of learning, then the question arises as to where the requisite cross-modality matching might occur. The olfactory cortex does not receive afferents from, or send efferents to, sensory neocortex. Each of the sensory cortices projects to the striatal complex, but recent anatomical studies indicate that the target areas within that structure are segregated from each other; for example, olfactory tubercle to ventral striatum (Heimer, 1978) and visual cortex to dorsal striatum (Nauta, 1979). Complex interactions involving frontal cortex and striatum (Goldman and Nauta, 1977; Groenewegen et al., 1990) might potentially provide the bases for cross-modal associations, but the neuroanatomical description of the relevant circuitries is not sufficiently precise to develop this idea into a more specific hypothesis. A perhaps more likely site for olfactory/nonolfactory comparisons is the amygdala. This region receives input from sensory neocortex via the perirhinal cortex and from olfactory cortex via its periamygdaloid extension (Ottersen, 1982; Amaral et al., 1992). It is thus conceivable that an odor influences the response of the amygdala to a subsequent visual input, and it will be of interest to test if damage to perirhinal or periamygdaloid cortex disrupts odor-object associations.

The final experiment showed that rats are able to integrate the long-term memory of odor-object associations with the transient memory of choices already made. The three control rats that came closest to mastering this task organized their selections according to fixed sequences. One factor that entered into this was the probability of making the correct object choice for a particular odor; that is, higher probability odors were selected before low probability ones (Fig. 7). Presumably then even relatively small differences in success rates have a lasting influence on the organization of olfactory behavior. Odors already sampled and responded to also influenced subsequent choices; that is, the rats made a different second selection depending on whether walnut- or chocolate-odor was the first choice (Fig. 8C). This may relate to the different foods associated with each odor in that the taste of a food item could influence the processing of a subsequent odor. Because of its interconnections with gustatory regions in brainstem and with hypothalamus (Burton and Benjamin, 1971; Bernard et al., 1993) the amygdala seems a likely site to mediate the influences of reward probability and food tastes on odor selections.

Two rats with entorhinal lesions performed well above chance in the serial odor-object association problem despite committing numerous reentry errors, confirming that encoding and use of associations between odors and objects can still be accomplished after cutting the major olfactory input to hippocampus. However, even though the above subset of lesioned animals were able to accomplish long series of correct choices, they succeeded in doing so at a reduced rate compared to the three control rats that came close to mastering the final task (see Fig. $6 A$ ). It is not unlikely that the poorer performance of the lesioned rats reflects their greater tendency to make reentry errors. Such errors could have an effect on arousal and other state variables known to affect the quality of performance in difficult tasks.

In summary, the present results suggest that the entorhinal cortex-hippocampal system encodes or prompts the encoding of transient memories concerned with recent choices; this memory system was not dependent upon cue configuration and if, as seems likely, is used in spatial mazes, then it would also be independent of sensory modality. While evidence indicative of hippocampal contributions to long-term olfactory memory was not obtained, it is certainly possible that the paradigms used in the studies reported here were not appropriate for detecting such contributions. Relationships between distant (e.g., extramaze, spatial) cues figure prominently in the physiological and behavioral literatures (e.g., O'Keefe and Conway, 1978; Muller et al., 1987) on hippocampus, but were not essential to correct performance in the present experiments. Accordingly, it would be of interest to test if rats are able to quickly form associations between odors and spatial locations or distant objects and, if so, if memory for such relationships is disrupted by lesions to hippocampus. Studies of this kind would have to be designed using behaviors that do not depend upon the transient memory system as, for example, is the case for performance in radial mazes.

The idea that hippocampus encodes both transient and longterm forms of memory finds indirect support in work showing that the structure contains more than one type of synaptic plasticity. Specifically, short bursts of stimulation delivered repeatedly to the Schaffer-commissural pathways induce long-term potentiation that is stable for days or even weeks (Staubli and Lynch, 1987, 1990); equivalent bursts given in pairs produce a weaker potentiation effect that decays over a period of hours and is absent at $24 \mathrm{hr}$ (Staubli et al., in press). This latter effect is a not unlikely substrate for the type of transient memory described here, while the much more stable LTP is suggestive of a very long lasting encoding of information. Recent studies have also provided evidence that potentiation in the mossy fiber synapses involves induction and expression processes that have little in common with LTP (Staubli et al., 1990; Zalutsky and Nicoll, 1990, 1992; Staubli, 1992). The presence of multiple plasticities prompts the argument that the hippocampus supports multiple forms of memory. Hopefully, it will be possible to devclop more spccific versions of this general hypothesis using variants of the behavioral designs employed in the present experiments.

\section{References}

Amaral DG, Price JL, Pitkaenen A, Carmichael ST (1992) Anatomical organization of the primate amygdaloid complex. In: The amygdala: neurobiological aspects of emotion, memory, and mental dysfunction (Aggleton JP, ed), pp 1-66. New York: Wiley-Liss.

Bernard JF, Alden M, Besson JM (1993) The organization of the efferent projections from the pontine parabrachial area to the amygdaloid complex: a Phaseolus vulgaris leucoagglutinin (PHA-L) study in the rat. J Comp Neurol 329:201-229.

Burton H, Benjamin RM (1971) Central projection of the gustatory system. In: Chemical senses, handbook of sensory physiology, Vol IV (Beidler LM, ed), pp 148-164. Berlin: Springer.

Cotman CW, Matthews DA, Taylor D, Lynch G (1973) Synaptic rearrangement in the dentate gyrus: histochemical evidence of adjustments after lesions in immature and adult rats. Proc Natl Acad Sci USA 70:3473-3477.

Gallagher M, King RA, Young NB (1983) Opiate antagonists improve spatial memory. Science 221:975-976.

Goldman PS, Nauta WJH (1977) An intricately patterned prefrontocaudate projection in the rhesus monkey. J Comp Neurol 171:369385.

Groenewegen HJ, Becker NE, Lohman AH (1980) Subcortical afferents of the nucleus accumbens septi in the cat, studied with retrograde axonal transport of horseradish peroxidase and bisbenzimide. Neuroscience 5:1903-1916.

Groenewegen HJ, Berendse HW, Wolters JG, Lohman AH (1990) The anatomical relationship of the prefrontal cortex with the striatopal- 
lidal system, the thalamus and the amygdala: evidence for a parallel organization. Prog Brain Res 85:95-116.

Heimer L (1978) The olfactory cortex and the ventral striatum. In: Limbic mechanisms (Livingston $\mathrm{M}$, Hornykiewicz $\mathrm{H}$, eds), pp 95187. New York: Plenum.

Hjorth-Simonsen A (1972) Projections of the lateral part of the entorhinal area to the hippocampus and fascia dentata. J Comp Neurol 146:219-232.

Jarrard LE, Okaichi H, Steward O, Goldschmidt RB (1984) On the role of hippocampal connections in the performance of place and cue tasks: comparisons with damage to hippocampus. Behav Neurosci 98:946-954.

Jung MW, Larson J, Lynch G (1990) Long-term potentiation of monosynaptic EPSPs in rat piriform cortex. Synapse 6:279-283.

Kanter ED, Haberly LB (1990) NMDA-dependent induction of longterm potentiation in afferent and association fiber systems of piriform cortex in vitro. Brain Res 525:175-179.

Kelley AE, Domesick VB, Nauta WJH (1982) The amygdalostriatal connections projection in the rat - an anatomical study by anterograde and retrograde tracing methods. Neuroscience 7:613-650.

Lynch G, Staubli U (1991) Possible contributions of long-term potentiation to the encoding and organization of memory. Brain Res Rev 16:204-206.

Lynch G, Matthews DA, Mosko S, Parks T, Cotman C (1972) Induced acetylcholinesterase-rich layer in rat dentate gyrus following entorhinal lesions. Brain Res 42:311-318.

Lynch G, Larson J, Staubli, U, Granger R (1991) Variants of synaptic potentiation and different types of memory operations in hippocampus and related structures. In: Memory: organization and locus of change (Squire LR, Weinberger NM, Lynch G, McGaugh J, eds), pp 330-363. New York: Oxford UP.

Macrides F, Eichenbaum HB, Forbes WB (1982) Temporal relationship between sniffing and the limbic theta rhythm during odor discrimination reversal learning. J Neurosci 2:1705-1717.

Muller RU, Kubie J, Ranck JB Jr (1987) Spatial firing patterns of hippocampal complex-spike cells in a fixed environment. J Neurosci 7:1935-1950.

Nauta WJH (1979) A proposed conceptual reorganization of the basal ganglia and telencephalon. Neuroscience 4:1875-1881.

O'Keefe JA, Conway DH (1978) Hippocampal place cells in the freely moving rat: why they fire where they fire. Exp Brain Res 31:573-590.

O'Keefe J, Nadel L (1978) The hippocampus as a cognitive map. London: Clarendon.

Olton DS, Feustle WA (1981) Hippocampal function required for nonspatial working memory. Exp Brain Res 41:380-389.

Olton DS, Becker JT, Handelmann GE (1979) Hippocampus, space and memory. Brain Behav Sci 2:313-365.

Ottersen OP (1982) Connections of the amygdala of the rat. IV. Corticoamygdaloid and intraamygdaloid connections as studied with axonal transport of horseradish peroxidase. J Comp Neurol 205:30-48.

Otto T, Eichenbaum H (1992) Complimentary roles of the orbital prefrontal cortex and the perirhinal-entorhinal cortices in an odorguided delayed-nonmatching-to-sample task. Behav Neurosci 106: 762-775.
Raffaele KC, Olton DS (1988) Hippocampal and amygdaloid involvement in working memory for nonspatial stimuli. Behav Neurosci 102: 349-355.

Ranck JB Jr (1973) Studies on single neurons in dorsal hippocampal formation and septum in unrestrained rats. Exp Neurol 41:461-555.

Rasmussen M, Barnes CA, McNaughton BL (1989) A systematic test of cognitive mapping, working-memory, and temporal discontiguity theories of hippocampal function. Psychobiology 17:335-348.

Roman F, Staubli U, Lynch G (1987) Evidence for synaptic potentiation in a cortical network during learning. Brain Res 418:221-226.

Schenk F, Morris RGM (1985) Dissociation between components of spatial memory in rats after recovery from the effects of retrohippocampal lesions. Exp Brain Res 48:11-28.

Staubli U (1992) A peculiar form of potentiation in mossy fiber synapses. In: Epilepsy research, Suppl, The dentate gyrus and its role in seizurcs (Ribak C, Gall CM, Mody I, eds), pp 151-157. Amsterdam: Elsevier.

Staubli U, Lynch G (1987) Stable hippocampal long-term potentiation elicited by 'theta' pattern stimulation. Brain Res 435:227-234.

Staubli U, Lynch G (1990) Stable depression of potentiated synaptic responses in the hippocampus with $1-5 \mathrm{~Hz}$ stimulation. Brain Res 13:113-118.

Staubli U, Ivy G, Lynch G (1984) Hippocampal denervation causes rapid forgetting of olfactory information in rats. Proc Natl Acad Sci USA 81:5885-5887.

Staubli U, Fraser D, Kessler M, Lynch G (1986) Studies on retrograde and anterograde amnesia of olfactory memory after denervation of the hippocampus by entorhinal cortex lesions. Behav Neural Biol 46: $432-444$.

Staubli U, Frascr F, Faraday R, Lynch G (1987) Olfaction and the "data" memory system in rats. Behav Neurosci 101:757-765.

Staubli U, Thibault O, DiLorenzo M, Lynch G (1989) Antagonism of NMDA receptors impairs acquisition but not retention of olfactory memory. Behav Neurosci 103:54-60.

Staubli U, Larson J, Lynch G (1990) Mossy fiber potentiation and long-term potentiation involve different expression mechanisms. Synapse 5:333-335.

Staubli U, Rogers G, Lynch G (1994) Facilitation of glutamate receptors enhances memory. Proc Natl Acad Sci USA 91:777-781.

Staubli U, Perez Y, Xu F, Rogers G, Ingvar H, Stone-Elander S, Lynch $G$ (in press) Centrally active modulators of glutamate (AMPA) receptors facilitate the induction of LTP in vivo. Proc Natl Acad Sci USA, in press.

Witter MP (1993) Organization of the entorhinal-hippocampal system: a review of current anatomical data. Hippocampus 3:33-44.

Youngentob SL, Markert LM, Mozell MM, Hornung DE (1990) A method for establishing a five odorant identification confusion matrix task in rats. Physiol Behav 47:1053-1059.

Youngentob SL, Markert LM, Hill TW, Matyas EP, Mozell MM (1991) Odor identification in rats: an update. Physiol Behav 49:1293-1296.

Zalutsky RA, Nicoll R (1990) Comparisons of two forms of long-term potentiation in single hippocampal neurons. Science 248:1619-1624.

Zalutsky RA, Nicoll RA (1992) Mossy fiber long-term potentiation shows specificity but no apparent cooperativity. Neurosci Lett 138 : 193-197. 